\title{
Effect of the Kinematic Lower Boundary Condition on the Spectral and Autocorrelation Structure of Annular Variability in the Troposphere
}

\author{
TORBEN KUnZ AND RichARD J. GREATBATCH \\ Helmholtz Centre for Ocean Research Kiel (GEOMAR), Kiel, Germany
}

(Manuscript received 3 April 2013, in final form 30 September 2013)

\begin{abstract}
The dynamical origin of the spectral and autocorrelation structure of annular variability in the troposphere is investigated by a deductive approach. Specifically, the structure of the power spectrum and autocorrelation function of the zonal-mean geopotential is analyzed for the case of a quasigeostrophic spherical atmosphere subject to a white noise mechanical forcing applied in a single Hough mode and concentrated at a particular level in the vertical, with vertically uniform Newtonian cooling and Rayleigh drag concentrated at a rigid lower boundary. Analytic expressions for the power spectrum are presented together with expressions for an approximate red noise (i.e., a Lorentzian-shaped) power spectrum. It is found that for an infinitely deep atmosphere the power spectrum can be well approximated by a red noise process for the first few Hough modes (associated with large Rossby heights), provided the distance from the forcing is not larger than about one Rossby height. When a frictional rigid lower boundary is included, however, the approximation is generally bad. The high-frequency part of the power spectrum exhibits near-exponential behavior and the autocorrelation function shows a transition from a rapid decay at short lags to a much slower decay at longer lags, if the thermal and mechanical damping time scales are sufficiently well separated. Since observed annular variability exhibits the same characteristics, the above results lead to the hypothesis that these characteristics may, to some extent, be intrinsic to the linear zonal-mean response problem-although the need for an additional contribution from eddy feedbacks is also implied by the results.
\end{abstract}

\section{Introduction}

Annular variability in the troposphere represents a major constituent of the large-scale atmospheric circulation and is associated with a considerable contribution to low-frequency variability on intraseasonal as well as interannual and longer time scales. It is often studied from either the zonal index (Lorenz and Hartmann 2003) or alternatively from the annular mode perspective (Thompson and Wallace 1998; sometimes also referred to as the Arctic or Antarctic Oscillation). Variability of the northern annular mode, for example, has been shown to have significant impacts on various aspects of weather and surface climate variability in the Northern Hemisphere as well as long-term trends in temperature and precipitation (e.g., Hurrell 1995; Thompson and Wallace 1998; Wallace 2000).

Corresponding author address: Torben Kunz, Helmholtz Centre for Ocean Research Kiel (GEOMAR), Düsternbrooker Weg 20, 24105 Kiel, Germany.

E-mail: tkunz@geomar.de
The basic dynamics of such annular variability are generally well understood. It is clear that such variability is intrinsically driven by eddy fluxes of heat and momentum, but feedbacks of the zonal flow on the eddies are also involved [see Lorenz and Hartmann (2003) and references therein]. However, external impacts are also suggested to play a role, such as modulations of the thermal forcing from below by variability of the oceanic component, or tropical and also stratospheric variability [see, e.g., Baldwin and Dunkerton (2001) and Hoerling et al. (2001), although some studies focus on the North Atlantic Oscillation, which, however, is closely related to the northern annular mode]. Concerning the predictability of annular variability on interannual and longer time scales, it is an important question whether these slow variations arise in response to slow external forcings, such as tropical sea surface temperatures, or simply reflect statistical sampling fluctuations due to unpredictable short-time-scale synoptic forcing. Different studies have provided different conclusions in this respect. Whereas Feldstein (2000), for example, suggests that virtually all interannual variability of the North Atlantic 
Oscillation can be interpreted as climate noise [see also Stephenson et al. (2000)], Keeley et al. (2009) conclude that a large fraction of the interannual variance should be expected to arise in response to slow external forcings.

These and related studies are always based on simple stochastic models against which the climate noise hypothesis may be tested. And usually it is assumed, probably for the sake of simplicity, that such atmospheric low-frequency variability can be described as an autoregressive, or red noise, process. On the other hand, it is found that neither the power spectra nor the autocorrelation functions (ACFs) of observed tropospheric annular variability have the corresponding simple structure (i.e., a Lorentzian-shaped power spectrum or an exponential ACF), but rather exhibit a distinct behavior on different time scales. This has been emphasized, in particular, by Osprey and Ambaum (2011). Specifically, the high-frequency part of the power spectrum is shown to exhibit near-exponential behavior and the associated $\mathrm{ACF}$ is composed of a rapid decay at lags of a few days and a much slower decay at longer lags. And the discrepancies in the conclusions regarding the climate noise hypothesis depend critically on whether these deviations from a red noise behavior are assumed a priori to be due to external forcings.

Hence, it is important to understand the origin of the abovementioned spectral and autocorrelation structure of annular variability in the troposphere, if one wants to assess the sources of its interannual and longer-timescale fluctuations. Whereas, for example, the red noise approach of Feldstein (2000) does not account for this distinct time-scale behavior at high frequencies, Keeley et al. (2009) assume that it must be due to slow external forcings. Another hypothesis is provided by Osprey and Ambaum (2011), who suggest deterministic low-order dynamical chaos may be responsible for the observed spectral and autocorrelation structure of tropospheric annular variability.

Given this inconclusiveness regarding the origin of the distinct spectral and autocorrelation structure, together with the importance of having a thorough understanding of this structure in view of the discussion above, the aim of this study is to clarify the nature of the spectral and autocorrelation structure of tropospheric annular variability from a dynamical perspective. To start, in this study, with the simplest aspect of the involved dynamical system, we focus exclusively on the dynamics of the zonal-mean circulation in response to a simple stochastic representation of midlatitude eddy fluxes and, therefore, explicitly neglect the effects of eddy-zonal flow feedbacks or any intrinsic properties of the eddy forcing itself. Specifically, we investigate the spectral and autocorrelation characteristics of the response of a zonal-mean quasigeostrophic (QG) spherical atmosphere, when subject to a white noise mechanical forcing, applied in a single Hough mode and concentrated at a particular level in the vertical, together with vertically uniform Newtonian cooling and Rayleigh drag concentrated at the lower boundary.

The basic equations for this approach are presented in section 2 [following the concepts of Plumb (1982), Garcia (1987), Haynes and Shepherd (1989), and Haynes et al. (1991)]. Subsequently, in section 3, we investigate the spectral and autocorrelation structure, separately for the case of an infinitely deep atmosphere, and the case with the additional effect of a frictional rigid lower boundary. The results are discussed in section 4 and conclusions are given in section 5 .

\section{Basic equations}

The approach to investigate the spectral and autocorrelation structure of annular variability is based on the equations that describe QG transformed Eulerian mean (TEM) flow, driven by zonally uniform thermal and mechanical forcing, in spherical and log-pressure coordinates, given by (e.g., Garcia 1987)

$$
\begin{aligned}
\bar{u}_{t}-f \bar{v}^{*} & =\bar{F}-\kappa \bar{u}, \\
f \bar{u}+a^{-1} \bar{\Phi}_{\phi} & =0, \\
-R H^{-1} \bar{T}+\bar{\Phi}_{z} & =0, \\
\bar{T}_{t}+N^{2} H R^{-1} \bar{w}^{*} & =\bar{Q}-\alpha \bar{T}, \\
(a \cos \phi)^{-1}\left(\bar{v}^{*} \cos \phi\right)_{\phi}+\rho_{0}^{-1}\left(\rho_{0} \bar{w}^{*}\right)_{z} & =0,
\end{aligned}
$$

where zonal averaging is denoted by overbars, $\bar{u}$ is the zonal wind, $\bar{v}^{*}$ and $\bar{w}^{*}$ are the northward and upward TEM residual velocities, $\bar{T}$ is temperature, and $\bar{\Phi}$ is geopotential. The nonconservative terms on the rhs of (1) and (4) represent the mechanical and thermal forcing, respectively, where $\bar{F}$ and $\bar{Q}$ are external forcings, $\kappa(z)$ is a Rayleigh drag coefficient, and $\alpha(z)$ is a Newtonian cooling coefficient. Hereafter, the above dependent variables shall represent anomalies from a suitable basic state. Also, $f=2 \Omega \sin \phi$ is the Coriolis parameter, $\Omega$ is the angular frequency, and $a$ the radius of Earth; $N^{2}(z)=R / H\left(T_{0 z}+k T_{0} / H\right)$ is the square of the buoyancy frequency, with a reference temperature profile $T_{0}(z)$, and $k=R / c_{p}$, where $R$ is the gas constant for dry air and $c_{p}$ is the heat capacity at constant pressure. A reference density profile is given by $\rho_{0} \propto \exp (-z / H)$. The meridional and vertical coordinates are latitude $\phi$ 
and $\log$-pressure height $z=-H \ln \left(p / p_{0}\right)$, respectively, with the density scale height $H$ and a reference pressure $p_{0}$. Partial derivatives with respect to $\phi, z$, and time $t$ are indicated by subscripts. For zero mechanical and thermal forcing, (1)-(5) represent the conservation laws for eastward, northward, and upward momentum; for thermodynamic energy; and for mass, respectively.

These equations can be combined into an elliptic partial differential equation for the zonal-mean geopotential tendency,

$$
\frac{N^{2}}{4 \Omega^{2} a^{2}} \mathcal{L}_{\phi} \Phi_{t}+\mathcal{L}_{z} \Phi_{t}=\frac{N^{2}}{4 \Omega^{2} a^{2}} \mathcal{L}_{\phi} F_{*}-\alpha \mathcal{L}_{z} \Phi
$$

with the second-order linear differential operators

$$
\begin{aligned}
& \mathcal{L}_{\phi}(\cdot)=\frac{1}{\sqrt{\cos \phi}} \frac{\partial}{\partial \phi}\left\{\frac{\cos \phi}{\sin ^{2} \phi} \frac{\partial}{\partial \phi}\left[\frac{1}{\sqrt{\cos \phi}}(\cdot)\right]\right\}, \\
& \mathcal{L}_{z}(\cdot)=\frac{1}{\sqrt{\rho_{0}}} \frac{\partial}{\partial z}\left\{\rho_{0} \frac{\partial}{\partial z}\left[\frac{1}{\sqrt{\rho_{0}}}(\cdot)\right]\right\},
\end{aligned}
$$

and where the modified variables

$$
\Phi=\mu \bar{\Phi}, \quad F_{*}=\mu \bar{F}_{*}
$$

have been used, with $\mu=\sqrt{\cos \phi \rho_{0}}$, and where $\bar{F}_{*}$ is related to $\bar{F}$ by the geostrophic wind relation (2) as $\bar{F}=-(a f)^{-1} \bar{F}_{* \phi}$. To allow for analytic progress below, we also assume an atmosphere with constant static stability, $N^{2}=$ const, and uniform Newtonian cooling, $\alpha=$ const. Additionally, since we are interested only in the response to mechanical external forcing, we set $Q=0$. For now, we also set $\kappa=0$, although Rayleigh drag will be included later in this section together with a rigid lower boundary to mimic the effect of an atmospheric boundary layer.

Following Garcia (1987), who derived an analogous equation for $\bar{w}^{*},(6)$ is separable, and solutions may be written as

$$
\Phi(\phi, z, t)=\sum_{n} \Phi^{(n)}(z, t) \Theta^{(n)}(\phi)
$$

Then, if the forcing is expanded in a similar way,

$$
F_{*}(\phi, z, t)=\sum_{n} F_{*}^{(n)}(z, t) \Theta^{(n)}(\phi),
$$

and, additionally, we seek oscillatory solutions

$$
\Phi^{(n)}(z, t)=\hat{\Phi}^{(n)}(z) \exp (i \omega t)
$$

in response to an oscillatory forcing that is concentrated at a particular level, say $z=0$ [as in Haynes et al. (1991)],

$$
F_{*}^{(n)}(z, t)=\hat{f}_{*}^{(n)} \delta(z / H) \exp (i \omega t)
$$

one obtains, by substitution of (10) and (11) into (6), a homogeneous horizontal structure equation,

$$
\mathcal{L}_{\phi} \Theta^{(n)}=\epsilon^{(n)} \Theta^{(n)},
$$

and a forced vertical structure equation,

$$
\mathcal{L}_{z} \hat{\Phi}^{(n)}-\gamma^{(n)} \hat{\Phi}^{(n)}=-\gamma^{(n)} /(i \omega) \hat{f}_{*}^{(n)} \delta(z / H),
$$

with

$$
\gamma^{(n)}=\frac{1}{H_{R}^{2}}\left(\frac{i \omega}{i \omega+\alpha}\right)
$$

where

$$
\frac{1}{H_{R}^{2}}=-\epsilon^{(n)} \frac{N^{2}}{4 \Omega^{2} a^{2}},
$$

and where $H_{R}$ is the Rossby height associated with the horizontal length scale $2 \pi a\left(-\epsilon^{(n)}\right)^{-1 / 2}$. As shown by Plumb (1982), the horizontal eigenvalues $\epsilon^{(n)}$ are all negative and the associated eigenfunctions $\Theta^{(n)}$ represent the orthonormal ${ }^{1}$ Hough modes (e.g., LonguetHiggins 1968; Andrews et al. 1987), which satisfy the lateral boundary conditions $\Theta_{\phi}^{(n)}=0$ at $\phi= \pm \pi / 2$. The symbol $\delta(\cdot)$ denotes the Dirac delta function.

The solutions to the vertical structure equation [(15)] in the homogeneous region, above or below the forcing, are of the form

$$
\hat{\Phi}^{(n)}=\hat{\Phi}_{0}^{(n)} \exp \left( \pm \Lambda^{(n)} z\right)
$$

where

$$
\Lambda^{(n)}=\left(\gamma^{(n)}+\frac{1}{4 H^{2}}\right)^{1 / 2}
$$

Since the coefficient $\gamma^{(n)}$ is complex, these solutions represent damped waves in the vertical. The fact that the

\footnotetext{
${ }^{1}$ In our case where we use the latitude $\phi$ [rather than the sine of latitude, as used by, e.g., Plumb (1982)] as the meridional coordinate, orthogonality follows from having included the $\sqrt{\cos \phi}$ factor in the modified variables, as defined by (9).
} 
upward- and downward-decaying solutions have the same vertical decay rate $\Lambda$ results from having included the $\rho_{0}^{1 / 2}$ factor in the modified variables, as defined by (9). The vertical asymmetry due to the density stratification of the atmosphere can be recovered at any time by dividing the solution by this factor. For a further discussion of the solutions (18), see Garcia (1987). In the following, our analysis will be concerned with the case in which the forcing consists of only a single Hough mode. Since the system is linear and thus the response is also limited to the same horizontal mode, the modal index $n$ can be dropped for brevity hereafter.

In the case of an infinitely deep atmosphere, the upper and lower boundary conditions are given by $\sqrt{\rho_{0}} \hat{\Phi} \rightarrow 0$ as either $z \rightarrow-\infty$ or $z \rightarrow+\infty$, respectively, because the finite angular momentum input, associated with $\hat{f}_{*}$, can produce only a finite angular momentum response. Thus, the solution to the forced vertical structure equation (i.e., the Green's function response to the mechanical forcing in a single Hough mode, concentrated at one particular level at $z=0$ ) consists of an upward-decaying component above (at $z>0$ ) and a downward-decaying component below the forcing (at $z<0$ ), where the Green's function coefficient

$$
\hat{\Phi}_{0}=\frac{H \gamma}{2 \Lambda} \frac{\hat{f}_{*}}{i \omega}
$$

is obtained by integration of (15) across $z=0$ and by requiring continuity of the geopotential response.

In the presence of a rigid lower boundary located at some level $z_{b}<0$ below the forcing, an upward-decaying solution $\hat{\Phi}_{B} \exp (-\Lambda z)$ is also possible at $z<0$ because the amount of angular momentum associated with this component is bounded in this case because of the finite depth of the layer between the forcing and the lower boundary, where $\hat{\Phi}_{B}$ represents the respective Green's function coefficient of this additional component, which reflects precisely the effect of the lower boundary on the solution. Thus, the Green's function response can now be expressed as

$$
\hat{\Phi}=\left\{\begin{array}{lll}
\hat{\Phi}_{0} \exp (-\Lambda z)+\hat{\Phi}_{B} \exp (-\Lambda z) & \text { at } & z>0 \\
\hat{\Phi}_{0} \exp (+\Lambda z)+\hat{\Phi}_{B} \exp (-\Lambda z) & \text { at } & z<0
\end{array} .\right.
$$

Additionally, we may assume a Rayleigh drag concentrated at the lower boundary by choosing $\kappa(z)=$ $\kappa_{b} \delta\left[\left(z-z_{b}\right) / H\right]$ on the rhs of the momentum equation [(1)]. For a detailed discussion of the Green's function response problem in the presence of a rigid lower boundary, see Haynes and Shepherd (1989); for the inclusion of boundary layer friction, see Haynes et al. (1991). In brief, the solution to the problem with a frictional rigid lower boundary is obtained as follows. First, the kinematic lower boundary condition is given by (Andrews et al. 1987; assuming a flat bottom)

$$
\bar{\Phi}_{t}+\frac{R T_{0 b}}{H} \bar{w}=0 \text { at } z=z_{b},
$$

where $\bar{w}$ is the Eulerian zonal-mean vertical velocity, which differs from the TEM residual $\bar{w}^{*}$ by the eddy heat flux term. However, if we assume, for simplicity, that eddy heat fluxes vanish at the lower boundary, we have $\bar{w}=\bar{w}^{*}$ at $z=z_{b}$ and we may still apply (22) to our problem. Then, by substitution of $\bar{u}$ by $\bar{\Phi}$ in the zonal momentum equation [(1)] through the geostrophic relation [(2)], use of the continuity equation [(5)], the horizontal structure equation [(14)], and the lower boundary condition [(22)], subsequent integration from $z_{b}$ to $+\infty$, and again requiring continuity of the geopotential response, we obtain the Green's function coefficient of the boundary component,

$$
\hat{\Phi}_{B}=B \exp \left(2 \Lambda z_{b}\right) \hat{\Phi}_{0},
$$

with

$$
B=\frac{\Lambda+(2 H)^{-1}-\gamma\left(M^{2}+\kappa_{b} / i \omega\right) H}{\Lambda-(2 H)^{-1}+\gamma\left(M^{2}+\kappa_{b} / i \omega\right) H},
$$

and where

$$
M^{2}=\frac{4 \Omega^{2} a^{2}}{-\epsilon} \frac{1}{R T_{0 b}},
$$

and $T_{0 b}=T_{0}\left(z_{b}\right)$. Then, the vertical geopotential Green's function response to the applied mechanical forcing at frequency $\omega$ may be written as

$$
\hat{\Phi}=\left\{\begin{array}{lll}
B_{\times}^{>} \hat{\Phi}_{0} \exp (-\Lambda z) & \text { at } & z>0 \\
B_{\times}^{<} \hat{\Phi}_{0} \exp (+\Lambda z) & \text { at } & z<0
\end{array},\right.
$$

where

$$
B_{\times}^{>}=B \exp \left(2 \Lambda z_{b}\right)+1
$$

and

$$
B_{\times}^{<}=B \exp \left[2 \Lambda\left(z_{b}-z\right)\right]+1 .
$$

From (23), or likewise from (27) and (28), it can be seen that, as $z_{b} \rightarrow-\infty$, the effect of the lower boundary 
vanishes, since $\hat{\Phi}_{B} \rightarrow 0$ in this case, and thus the solution for an infinitely deep atmosphere is recovered.

\section{Power spectra and autocorrelation functions}

With the solutions (26) presented in the previous section, we can now proceed to investigate the associated power spectra and autocorrelation functions of the zonally averaged geopotential that arise in response to a white noise mechanical forcing applied at a particular level. Below, we will first consider the case of an infinitely deep atmosphere, subsequently include the effect of a frictional rigid lower boundary, and, finally, we will illustrate the implications of the obtained results for annular variability in the troposphere.

\section{a. Infinitely deep atmosphere}

In the case of an infinitely deep atmosphere, the solutions (26) are symmetric above and below the forcing and, thus, it is sufficient to consider only the upward branch, for instance. The power spectrum $S(\omega ; z)$ of the zonally averaged geopotential at a given height is obtained as the squared absolute value of $\hat{\Phi}$, assuming a white noise mechanical forcing (i.e., $\left|\hat{f}_{*}(\omega)\right|=$ const) with power spectrum $\left|\hat{f}_{*}\right|^{2}$,

$$
S(\omega ; z)=\left|\hat{\Phi}_{0}\right|^{2}|\exp (-\Lambda|z|)|^{2} .
$$

As a first step, we shall analyze the structure of the local spectrum $S(\omega ; z=0)=\left|\hat{\Phi}_{0}\right|^{2}$ at the level of the forcing, which can be written as

$$
\begin{aligned}
S(\omega ; z=0) & =\left|\frac{H \gamma}{2 \Lambda} \frac{\hat{f}_{*}}{i \omega}\right|^{2} \\
& =\frac{c}{4}\left[\frac{\left|\hat{f}_{*}^{2}\right|}{1+(4 c)^{-1}}\right]\left[\left(\omega^{2}+\alpha_{L}^{2}\right)+\frac{\omega \alpha}{1+(4 c)^{-1}}\right]^{-1},
\end{aligned}
$$

with $c=H^{2} / H_{R}^{2}$, and where

$$
\alpha_{L}=\alpha(1+4 c)^{-1 / 2}
$$

If the Rossby height is large compared to the density scale height, then the second frequency-dependent term in (31) can be neglected [which is equivalent to neglecting the imaginary part of the argument of the root $\Lambda$ in the denominator of (30)], and the local spectrum [(31)] can be approximated by a red noise (i.e., a Lorentzianshaped) power spectrum $S_{L}$ as

$$
S_{L}(\omega)=\frac{2 \sigma_{L}^{2} \alpha_{L}}{\omega^{2}+\alpha_{L}^{2}},
$$

where

$$
\sigma_{L}^{2}=\frac{c}{4}\left[\frac{\left|\hat{f}_{*}^{2}\right|}{1+(4 c)^{-1}}\right] \frac{1}{2 \alpha_{L}}
$$

is the variance of the local red noise approximation, obtained by integration of the power spectrum from $-\infty$ to $+\infty$ [using the fact that $\int\left(\omega^{2}+\alpha_{L}^{2}\right)^{-1} d \omega=$ $\left.\left(2 \pi \alpha_{L}\right)^{-1} \arctan \left(\omega \alpha_{L}^{-1}\right)\right]$, and $\alpha_{L}$ can be identified as the local effective damping rate. Since the neglected term [i.e., the second frequency-dependent term in (31)] vanishes as $\omega \rightarrow 0$ and also becomes irrelevant compared to the first frequency-dependent term as $\omega \rightarrow \infty$, the deviation of the true spectrum from its red noise approximation maximizes at intermediate frequencies and vanishes in both the low- and high-frequency limits.

The true local spectrum and its red noise approximation are shown in Fig. 1a (top black and dashed red lines, respectively), for the first Hough mode $(n=1)$, for $\alpha^{-1}=20$ days, and for an isothermal atmosphere at $240 \mathrm{~K}$, implying $N^{2}=4 \times 10^{-4} \mathrm{~s}^{-2}$ and $H \approx 7 \mathrm{~km}$. Additionally, Fig. $1 \mathrm{~b}$ shows the corresponding ACFs (bottom black and dashed red lines, respectively). For the true solution, the ACF is obtained as the inverse Fourier transform of the corresponding spectrum (where the involved integral is done numerically), whereas for the red noise approximation the ACF is readily given as an exponential that decays at rate $\alpha_{L}$. It is seen that the local spectrum and ACF are well approximated by their corresponding red noise expressions, where the local ACF $e$-folding time scale is given by $\alpha_{L}^{-1}$, which, for $n=1$ and $N^{2}=4 \times 10^{-4} \mathrm{~s}^{-2}$ [corresponding to a horizontal length scale of $\left.L_{y}=2 \pi a(-\epsilon)^{-1 / 2} \approx 14000 \mathrm{~km}\right]$, is $\alpha_{L}^{-1}=1.32 \alpha^{-1}$. From (32) it is clear that the ratio $\alpha / \alpha_{L}$ is always greater than unity, which reflects the counteracting effect of the secondary circulation against the applied mechanical forcing due to the induced QG adjustment. Table 1 lists this ratio for the first six Hough modes together with the associated Rossby heights and horizontal length scales. For higher modes with smaller Rossby heights, this ratio increases monotonically and attains a value of 3.14 at $n=6$ (with $L_{y} \approx 4000 \mathrm{~km}$ ). However, for higher modes, the Rossby height becomes comparable to or even smaller than the density scale height and, thus, the deviation of the true spectrum from its red noise approximation increases. Figure 2a shows the local and the approximated power spectrum (top black and dashed red lines, respectively) for $n=4$ (with 

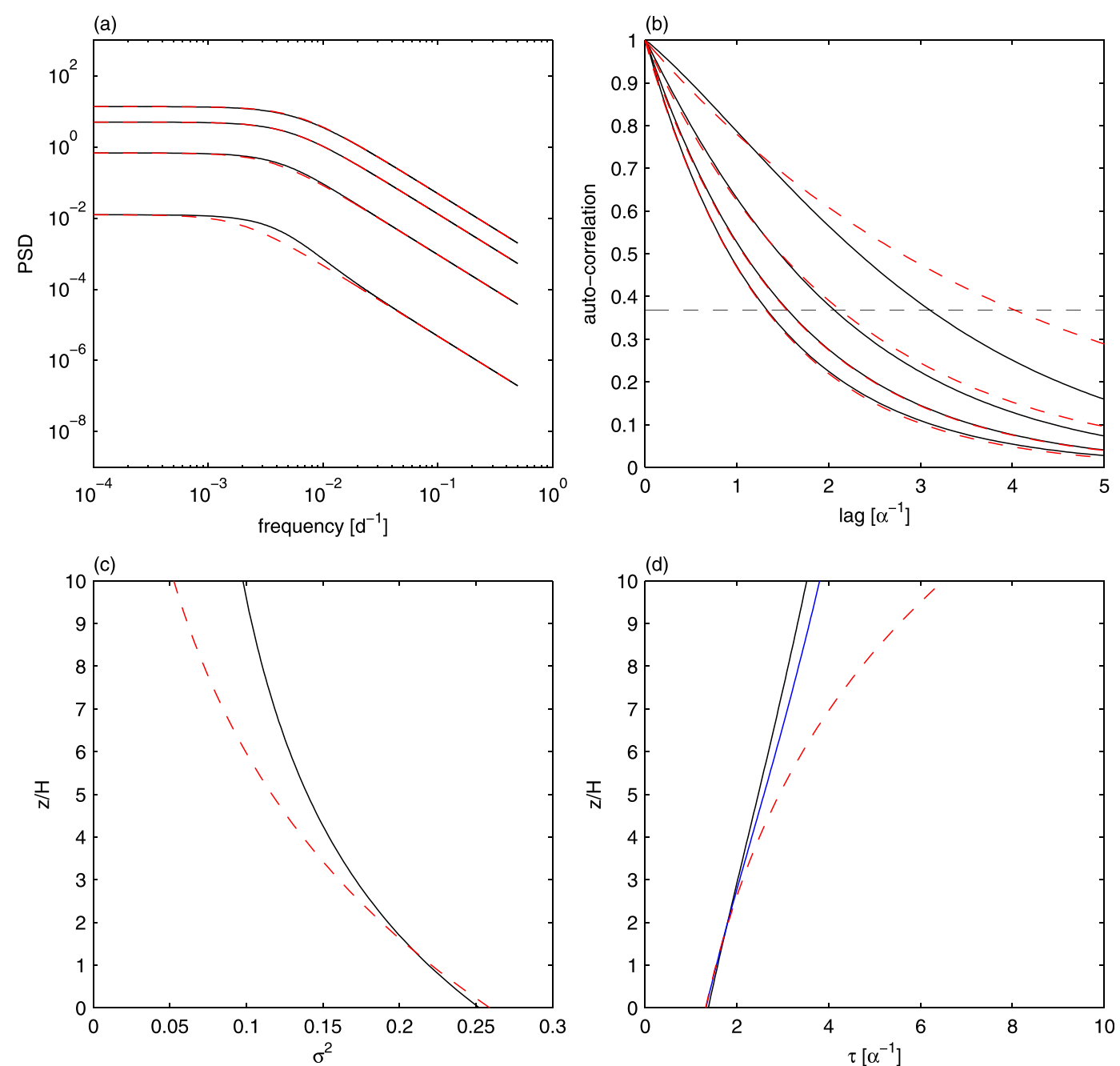

FIG. 1. (a) Power spectra (black) and their red noise approximations (dashed red) for the case of an infinitely deep atmosphere. (b) ACFs (black) and their red noise approximations (dashed red) for the same case. The horizontal dashed black line marks $e^{-1}$. Spectra and ACFs are shown for a distance away from the forcing of $z=0, H, 3 H, 7 H$, corresponding in (a) to the top to bottom pairs of curves and in (b) to the bottom to top pairs of curves. (c) Vertical profiles of the variance of the true spectra (black) and their red noise approximations (dashed red), divided by $\rho_{0}$. Units of variances and of the spectra are arbitrary owing to the linearity of the system. (d) Vertical profiles of the integrated memory (black), the $e$-folding time scale (blue), and its red noise approximation (dashed red). All quantities are shown for $n=1, \alpha^{-1}=20$ days, and for an isothermal atmosphere at $240 \mathrm{~K}$.

$\left.H_{R} \approx H\right)$, and the corresponding ACFs are presented in Fig. 2b (bottom black and dashed red lines, respectively). The red noise spectrum overestimates the variance at intermediate frequencies, and also the ACF changes its shape compared to an exponential decay, as given, for example, by the approximated red noise ACF.

Next, we analyze the structure of the remote spectrum at levels away from the forcing. The remote effect arises from the additional factor $E^{2}=|\exp (-\Lambda|z|)|^{2}$ in (29). To also find an approximate remote red noise spectrum $S_{R}$ that fits the true spectrum in both the low- and highfrequency limit, we need to approximate $E^{2}$ as

$$
E^{2} \approx G=A \frac{\omega^{2}+\alpha_{L}^{2}}{\omega^{2}+\alpha_{R}^{2}},
$$

with an amplitude factor $A$ and the effective damping rate $\alpha_{R}$ of the remote red noise approximation, chosen such that the limits of $G$,

$$
G \rightarrow G_{0}=A \alpha_{L}^{2} / \alpha_{R}^{2} \text { as } \omega \rightarrow 0
$$

and

$$
G \rightarrow G_{\infty}=A \text { as } \omega \rightarrow \infty,
$$


TABLE 1. Horizontal length scale $L_{y}=2 \pi a(-\epsilon)^{-1 / 2}(\mathrm{~km})$, Rossby height $H_{R}(\mathrm{~m})$, and the ratio $\alpha / \alpha_{L}$ for the first six Hough modes $(n=1, \ldots, 6)$, and an isothermal atmosphere at $240 \mathrm{~K}$.

\begin{tabular}{lrrr}
\hline$n$ & $L_{y}$ & $H_{R}$ & $\alpha / \alpha_{L}$ \\
\hline 1 & 14042 & 16297 & 1.32 \\
2 & 11304 & 13120 & 1.47 \\
3 & 6726 & 7807 & 2.06 \\
4 & 5985 & 6947 & 2.26 \\
5 & 4410 & 5119 & 2.92 \\
6 & 4072 & 4727 & 3.14 \\
\hline
\end{tabular}

are equal to the corresponding squared limits of $E$,

$E \rightarrow E_{0}=\exp \left[-|z|\left(\frac{1}{4 H^{2}}\right)^{1 / 2}\right]$ as $\omega \rightarrow 0$

and
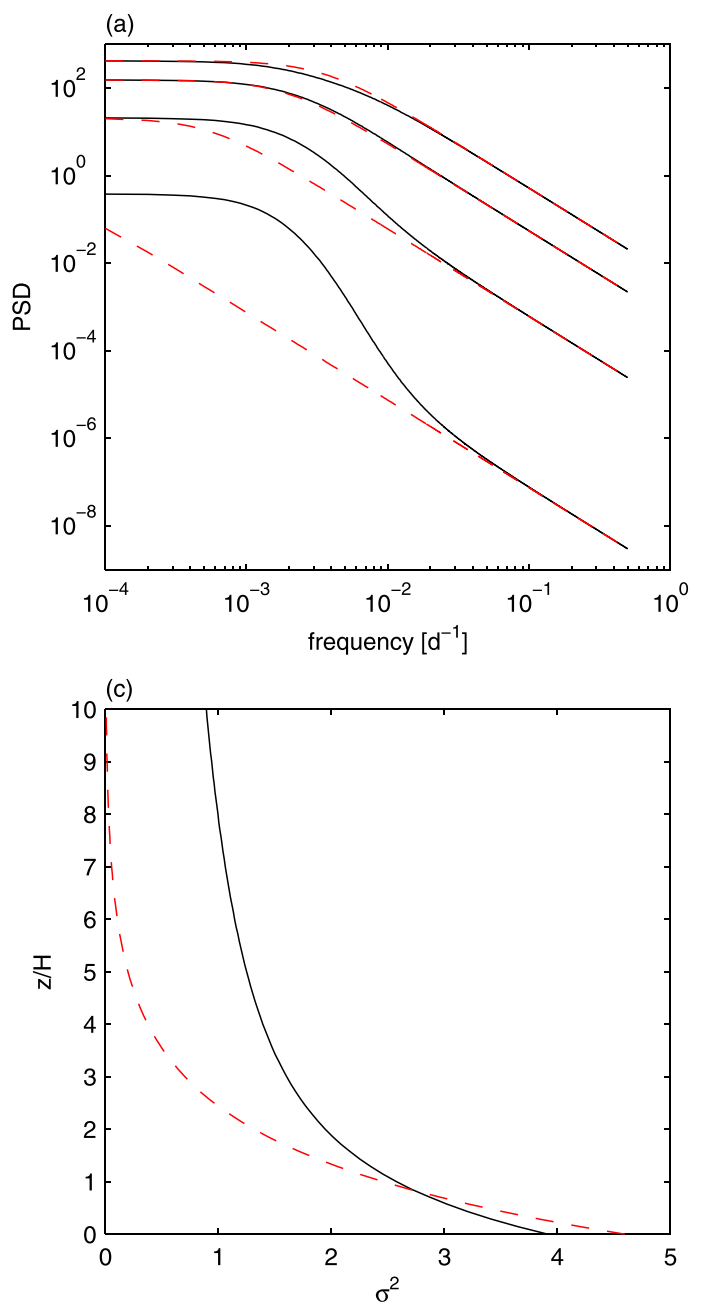

$E \rightarrow E_{\infty}=\exp \left[-|z|\left(\frac{1}{H_{R}^{2}}+\frac{1}{4 H^{2}}\right)^{1 / 2}\right]$ as $\omega \rightarrow \infty$

(i.e., by requiring $G_{\infty}=E_{\infty}^{2}$ and $\left.G_{0} / G_{\infty}=E_{0}^{2} / E_{\infty}^{2}\right)$. This implies

$\alpha_{R}(z)=\alpha_{L} E_{\infty} / E_{0}$

$$
=\alpha_{L} \exp \left\{-|z|\left[\left(\frac{1}{H_{R}^{2}}+\frac{1}{4 H^{2}}\right)^{1 / 2}-\frac{1}{2 H}\right]\right\}
$$

and $A=E_{\infty}^{2}$. Thus, the approximate remote red noise spectrum is given by

$$
S_{R}(\omega ; z)=\frac{2 \sigma_{R}^{2} \alpha_{R}}{\omega^{2}+\alpha_{R}^{2}}
$$

with the variance
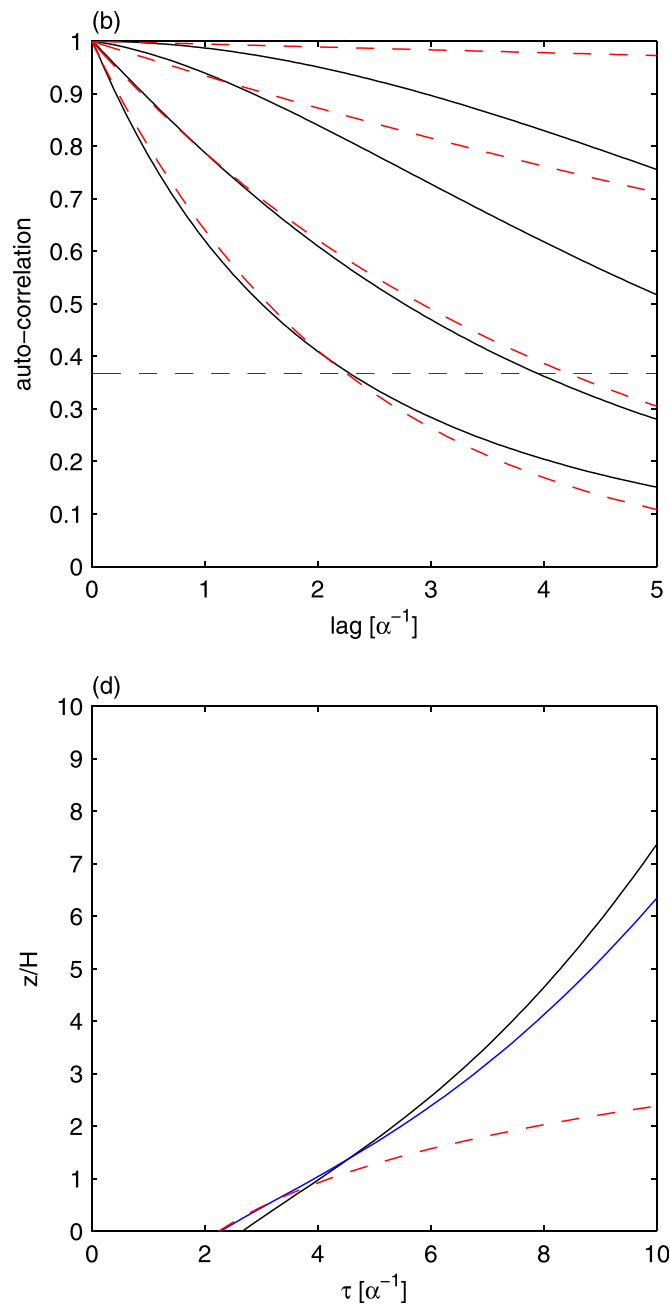

FIG. 2. As in Fig. 1, but for $n=4$. 


$$
\sigma_{R}^{2}(z)=\frac{c}{4} E_{\infty}^{2}\left[\frac{\left|\hat{f}_{*}^{2}\right|}{1+(4 c)^{-1}}\right] \frac{1}{2 \alpha_{R}} .
$$

The true remote spectra and their red noise approximations are shown in Fig. 1a (for $n=1$ ). At large distance away from the forcing, for example, at $z=7 \mathrm{H}$ (bottom black and dashed red lines, respectively), the red noise spectrum significantly underestimates the variance at intermediate frequencies. This vertically increasing mismatch is seen also in the vertical profile of the full variance $\sigma_{R}^{2}$ (divided by $\rho_{0}$ in order to recover the vertical asymmetry due to the density stratification of the atmosphere) in Fig. 1c (red dashed line), when compared to the full variance of the true remote spectrum (black line; obtained by numerical integration over the spectrum and again dividing by $\rho_{0}$ ). Since the width of the zero-frequency peak of the remote spectrum is underestimated by the red noise approximation, the corresponding ACF $e$-folding time scale shown in Fig. 1b (at $z=7 H$, for example; see top black and dashed red lines, respectively) is overestimated, consistently with the Fourier reciprocity principle, and also the shape of the ACF deviates from that of an exponential decay. The vertical profiles of the true ACF $e$-folding time scale $\tau_{e}$ and of its approximation $\alpha_{R}^{-1}$ are shown in Fig. 1d (blue and dashed red lines, respectively) together with the integrated memory $\tau_{m}$ (black line). The integrated memory is computed by numerical integration of the true ACF from lag 0 to $\infty$ and equals $\tau_{e}$ in case of an exponential ACF (i.e., for a red noise process). The fact that at large distances from the forcing these two time-scale measures increasingly deviate from each other (see Fig. 1d) expresses the increasing difference in shape of the ACF from an exponential decay. All these characteristics become even more pronounced for higher horizontal modes, as illustrated by Fig. 2 for $n=4$. Since, as mentioned above, the local red noise spectrum overestimates, but the remote red noise spectrum underestimates the variance at intermediate frequencies, in turns out (see Fig. 2a) that there is some intermediate distance from the forcing at which the true spectrum and its red noise approximation fit best, seen in the example for $n=4$ near $z=H$ (second black and dashed red lines from top, respectively).

In summary, the local power spectrum, the corresponding ACF, and its time-scale measures can be well approximated by a red noise process for the first few Hough modes. The remote spectra, ACFs, and time scales, however, can be approximated by a red noise process only over the first few scale heights for $n=1$ and over only one scale height for $n=4$. For higher modes, both the local and remote spectra fit increasingly less well with the red noise approximations (not shown). In the following, however, we do not further investigate the reasons for the deviations from red noise characteristics at large distances from the forcing, since we will finally be concerned with the implications for tropospheric variability that arises in response to tropospheric forcing and, thus, the domain of interest spans over only a little more than one scale height.

\section{b. Effect of the lower boundary}

We now turn to the effect of a frictional rigid lower boundary on the power spectra and ACFs. Since in this case $\hat{\Phi}_{B} \neq 0$, we need to include the terms defined in (27) or (28) in the power spectrum, which is then given by

$S(\omega ; z)=\left\{\begin{array}{lll}\left|B_{\times}^{>}\right|^{2}\left|\hat{\Phi}_{0}\right|^{2}|\exp (-\Lambda z)|^{2} & \text { at } & z>0 \\ \left|B_{\times}^{<}\right|^{2}\left|\hat{\Phi}_{0}\right|^{2}|\exp (+\Lambda z)|^{2} & \text { at } & z<0\end{array}\right.$.

It is of interest to see how well this spectrum can again be approximated by a red noise spectrum. This can be done in exactly the same way as for the remote spectrum in the previous subsection, but with the low- and highfrequency limits of $E$ replaced by the corresponding limits of $B_{\times}^{>}$or $B_{\times}^{<}$, respectively, given as

$$
B_{\times}^{>} \rightarrow B_{\times 0}^{>}=B_{0} \exp \left(\frac{z_{b}}{H}\right)+1 \quad \text { as } \omega \rightarrow 0
$$

and

$$
B_{\times}^{>} \rightarrow B_{\times \infty}^{>}=B_{\infty} \exp \left[2 z_{b}\left(\frac{1}{H_{R}^{2}}+\frac{1}{4 H^{2}}\right)^{1 / 2}\right]+1 \quad \text { as } \omega \rightarrow \infty
$$

for $z>0$, and by

$B_{\times}^{<} \rightarrow B_{\times 0}^{<}=B_{0} \exp \left(\frac{z_{b}-z}{H}\right)+1$ as $\omega \rightarrow 0$

and

$$
\begin{aligned}
B_{\times}^{<} \rightarrow B_{\times \infty}^{<}= & B_{\infty} \exp \left[2\left(z_{b}-z\right)\left(\frac{1}{H_{R}^{2}}+\frac{1}{4 H^{2}}\right)^{1 / 2}\right] \\
& +1 \text { as } \omega \rightarrow \infty
\end{aligned}
$$

for $z<0$, where $B_{0}$ and $B_{\infty}$ are the limits of $B$,

$$
B \rightarrow B_{0}=\frac{1}{c} \frac{\alpha}{\kappa_{b}}-1 \text { as } \omega \rightarrow 0,
$$


and

$$
B \rightarrow B_{\infty}=\frac{(c+1 / 4)^{1 / 2}+1 / 2-M^{2} c}{(c+1 / 4)^{1 / 2}-1 / 2+M^{2} c} \text { as } \omega \rightarrow \infty .
$$

The approximate red noise spectrum is then obtained as

$$
S_{B}(\omega ; z)=\frac{2 \sigma_{B}^{2} \alpha_{B}}{\omega^{2}+\alpha_{B}^{2}}
$$

with the effective damping rate

$$
\alpha_{B}(z)=\left\{\begin{array}{lll}
\alpha_{R}(z) B_{\times \infty}^{>} / B_{\times 0}^{>} & \text {at } & z>0 \\
\alpha_{R}(z) B_{\times \infty}^{<} / B_{\times 0}^{<} & \text {at } & z<0
\end{array}\right.
$$

and the variance

$$
\sigma_{B}^{2}(z)=\left\{\begin{array}{l}
\frac{c}{4} B_{\times \infty}^{>2} E_{\infty}^{2}\left[\frac{\left|\hat{f}_{*}^{2}\right|}{1+(4 c)^{-1}}\right] \frac{1}{2 \alpha_{B}} \text { at } z>0 \\
\frac{c}{4} B_{\times \infty}^{<2} E_{\infty}^{2}\left[\frac{\left|\hat{f}_{*}^{2}\right|}{1+(4 c)^{-1}}\right] \frac{1}{2 \alpha_{B}} \text { at } z<0
\end{array} .\right.
$$

The true power spectra and their red noise approximations in the presence of the lower boundary are shown in Fig. 3a for $\kappa_{b}^{-1}=1$ day and for the limit $z_{b} \rightarrow 0$ [for which the exponential terms in (45) and (46) become irrelevant]. It is obvious that the true spectrum is well approximated by the red noise spectrum only locally at $z=0$ (top black and dashed red lines, respectively), whereas already one scale height above the forcing (and, in this case, the boundary) the variance is significantly overestimated at intermediate frequencies (see also the variance profiles in Fig. 3c). The corresponding ACFs (Fig. 3b) also exhibit a strong change in shape once the lower boundary is introduced (when compared with Fig. 2b), with an abrupt transition between a fast decay at short lags and a slow decay at long lags, and the correlation at which the transition occurs increases with increasing distance from the forcing. This leads to a sudden jump with height of $\tau_{e}$ (see blue line in Fig. 3d), whereas $\tau_{m}$ (black line) increases continuously, which expresses the fact that the ACFs are by far not exponentials in this case. The shapes of the spectra and, in particular, of the ACFs suggest a superposition of a slow and a fast component.

To further investigate this aspect, we analyze the complex geopotential solution (26), for $z \geq 0$, directly rather than its power spectrum or ACF. The frequency dependence of the solution is illustrated in Figs. 4a-c for $\hat{f}_{*}=1$. At $z=0$ (Fig. 4a), the behavior reflects the almost perfect red noise characteristics as already seen in the power spectrum, with increasing phase lag behind the forcing with increasing frequency. However, with increasing distance above the forcing (Figs. $4 \mathrm{~b}$ and $4 \mathrm{c}$ ), the amplitude of this fast component reduces and a second component appears and increasingly dominates the overall solution, although the transition remains sharp, which reflects the step in the power spectrum at intermediate frequencies as the spectrum is the square of the distance in Fig. 4 from the origin of the complex plane. Furthermore, the sharpness of the transition is a function of the separation between the thermal and the mechanical damping time scales, with a smooth transition when $\alpha$ is close to $\kappa_{b}$ (not shown).

Figures $4 \mathrm{~d}-\mathrm{f}$ show the same geopotential solution but with the thermal damping parameter $\alpha$ set to zero. It is obvious that this thermally undamped component can be identified with the fast one of the two components of the full solution. It can be shown that the low-frequency limit of this thermally undamped component (in the limit $z_{b} \rightarrow 0$ considered here) is given as ${ }^{2}$

$\hat{\Phi}(z)=\frac{\hat{f}_{*}}{\kappa_{b}} \exp \left[-z\left(\frac{1}{H_{R}^{2}}+\frac{1}{4 H^{2}}\right)^{1 / 2}\right]$ as $\omega \rightarrow 0$.

Since, by contrast, the corresponding vertical decay rate $\Lambda$ of the full solution is always smaller than that of the solution (54), it is clear that the amplitude ratio between the thermally undamped component and the remainder decreases with height, as seen in Figs. 4a-c, but also in Fig. 3b. The narrow peak in the ACF that is focused on short lags becomes less important with increasing height, when compared to the slowly decaying component in the ACF at long lags, which reflects the low-frequency component in Figs. $4 \mathrm{~b}$ and $4 \mathrm{c}$. It is interesting to see that the two components that determine the characteristic structure of the power spectra and the ACFs in the presence of the lower boundary are not the two components seen in (21), but that they are (i) the thermally undamped component that reflects solely the interaction of the mechanical forcing with the boundary layer friction and (ii) the remaining component that reflects the interaction of the thermal damping of the induced anomalies with the boundary layer friction,

\footnotetext{
${ }^{2}$ It can also be shown that, in the limit $z_{b} \rightarrow 0$, the thermally undamped component behaves precisely like a red noise process with damping coefficient $\kappa_{1}=\kappa_{b}\left\{\left[1+(4 c)^{-1}\right]^{1 / 2}-1 / 2+M^{2}\right\}^{-1}$, corresponding to the power spectrum at $z=0$ shown in Fig. 3a (top black and dashed red lines, respectively).
} 

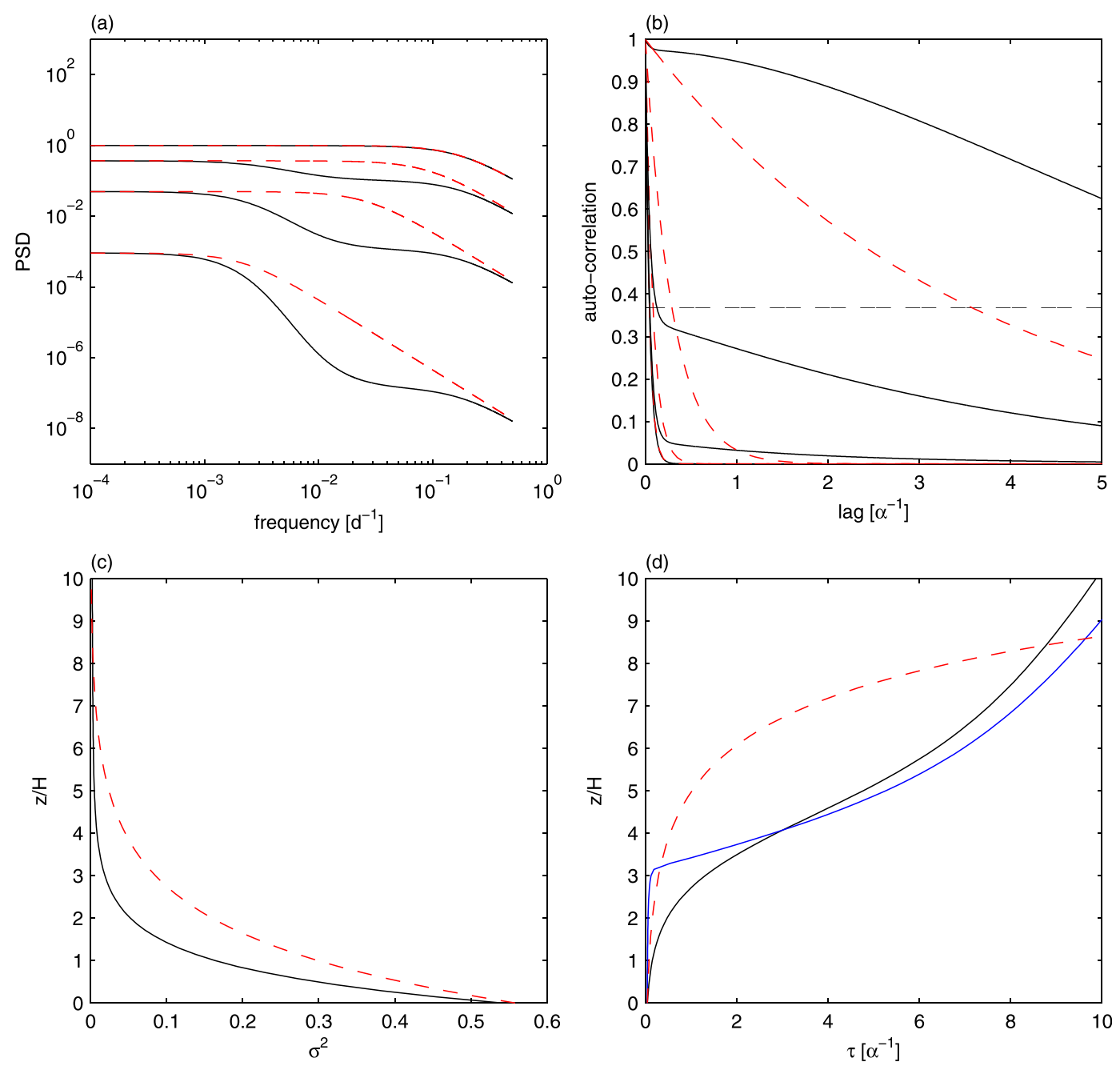

FIG. 3. As in Fig. 2, but for the case with a frictional rigid lower boundary at $z=0$.

where the QG adjustment of both components is modified through the rigid lower boundary.

So far we have considered only the limit $z_{b} \rightarrow 0$ because then the effects of the lower boundary are strongest and the solution is simpler because of the irrelevance of the exponential terms in (45) and (46). When the boundary is located below the forcing the principle behavior remains unchanged, but the effect of the boundary is weaker. Therefore, we only briefly present the case with $z_{b}=-3 H$. As can be seen from Figs. 5a and 5b, the behavior near and above the forcing is virtually unaffected by the lower boundary (when compared to the case of an infinitely deep atmosphere, shown in Fig. 2). And below the forcing, there is a gradual transition between the unbounded solution and the solution with the boundary, which leads to a maximum in the time scales at intermediate levels, but the variance there is very small according to the fast downward decay because of the density stratification. The red noise approximations appear again to be a rather bad fit to the true solutions in this case.

In summary, once a frictional rigid lower boundary is introduced, the power spectra and ACFs exhibit a dramatic change in their behavior and their characteristics deviate strongly from that of a red noise process. Moreover, the solution appears to consist of two superimposed components, both of which are influenced by the frictional rigid lower boundary, and one dominates the high-frequency behavior and the other one dominates the low-frequency behavior.

\section{c. Implications for annular variability in the troposphere}

The above analysis shows that, in the presence of a frictional rigid lower boundary, the ACF exhibits a clear transition between a fast decay at short lags and a slow 

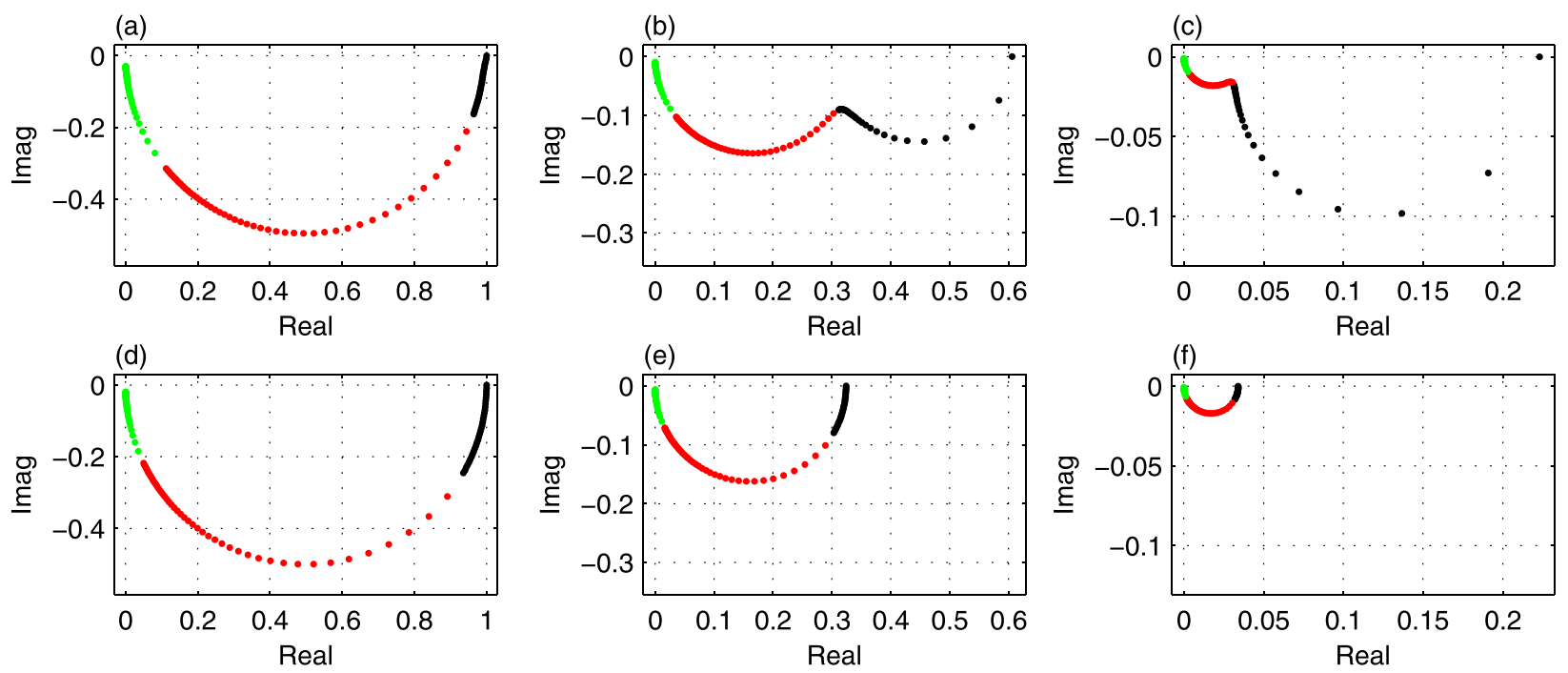

FIG. 4. (a)-(c) Geopotential Green's function response [see (26)] at (a) $z=0$, (b) $z=H$, and (c) $z=3 H$, as a function of frequency. The frequency range $\omega=2 \pi \times(0, \ldots, 0.03)$ at intervals of $\Delta \omega=2 \pi \times 0.001$ is shown by black dots, the range $\omega=2 \pi \times(0.04, \ldots, 0.5)$ at intervals of $\Delta \omega=2 \pi \times 0.01$ is shown by red dots, and the range $\omega=2 \pi \times(0.6, \ldots, 10)$ at intervals of $\Delta \omega=2 \pi \times 0.1$ is shown by green dots; frequencies are in units of day ${ }^{-1}$. (d)-(f) As in (a)-(c), but for the thermally undamped component of the response.

decay at long lags (as long as $\alpha$ and $\kappa_{b}$ are clearly separated). Since such a feature also occurs in the ACF of the northern annular mode [as shown by, for example, Osprey and Ambaum (2011)], it is plausible to investigate whether this effect of the lower boundary may explain the observed ACF structure of the northern annular mode. As a very simple idealized tropospheric setup, we consider the following case with now two forcings located at different levels. One forcing is located at $z=0$ and the second forcing at $z=H$ (both with the same amplitude); we set $H=8 \mathrm{~km}$ and $N^{2}=1.5 \times$ $10^{-4} \mathrm{~s}^{-2}$; and, as before, $\alpha^{-1}=20$ days, $\kappa_{b}^{-1}=1$ day, and $n=4$. The two forcings are thought to represent upperand lower-tropospheric eddy forcing, where the lowlevel forcing in reality is due to the divergence of the vertical EP-flux component inside the planetary boundary layer, since eddy heat fluxes are largely reduced at the bottom of this layer but maximize typically near its top. ${ }^{3}$

\footnotetext{
${ }^{3}$ Here we have to assume that eddy heat fluxes vanish completely at $z=0$ for the kinematic lower boundary condition [(22)] to be applicable. However, this does not reduce the plausibility of the lower-tropospheric eddy forcing in our idealized setup because the divergence of the vertical EP flux component would even slightly increase for vanishing surface eddy heat fluxes. The same simplification has been made in a related context by Brayshaw et al. (2009); see their appendix. Effectively, the $\delta$-function forcing at $z=0$ means that the total effect of near-surface eddy heat fluxes on the acceleration of the zonal flow across the boundary layer is concentrated within a thin layer just above the surface. See also Held and Schneider (1999) for an illustrative discussion of this issue.
}

Then we obtain the power spectra for this case as $S(\omega ; z)=\left|\hat{\Phi}_{1}(\omega ; z)+\hat{\Phi}_{2}(\omega ; z)\right|^{2}$ [where $\hat{\Phi}_{1}(\omega ; z)$ and $\hat{\Phi}_{2}(\omega ; z)$ represent the geopotential components that arise in response to the two forcings], implying the assumption that the two forcings were perfectly correlated in time. This is another oversimplification. However, when we assume that they are either fully uncorrelated, by setting $S(\omega ; z)=\left|\hat{\Phi}_{1}(\omega ; z)\right|^{2}+\left|\hat{\Phi}_{2}(\omega ; z)\right|^{2}$ (i.e., assuming zero covariance), or, alternatively, that they are perfectly anticorrelated, by setting $S(\omega ; z)=\left|\hat{\Phi}_{1}(\omega ; z)-\hat{\Phi}_{2}(\omega ; z)\right|^{2}$, the results are qualitatively similar (not shown). The variances, ACFs, and time scales are then obtained from these spectra as before.

The results, at $z=5 \mathrm{~km}$, are presented in Fig. 6 by the dashed red lines. For comparison, we also show all quantities for the case of an infinitely deep atmosphere by the dashed green lines. Figure 6a shows the expected change when introducing the lower boundary. The spectrum becomes flatter owing to the fast frictional damping associated with the boundary and, accordingly, the $e$-folding time scale is reduced (Fig. 6c), but also the shapes of the spectra and the ACFs change as described above; that is, the spectrum exhibits a smooth step at intermediate frequencies and the ACF decays rapidly at short lags and slowly at long lags. When the spectra are shown in semilog representation, as in Fig. 6b, we find that the high-frequency part of the spectrum also changes its characteristics from a Lorentzianshaped to a near-exponentially shaped structure. This is also of interest because this is another feature of observed northern annular mode variability (Osprey and 

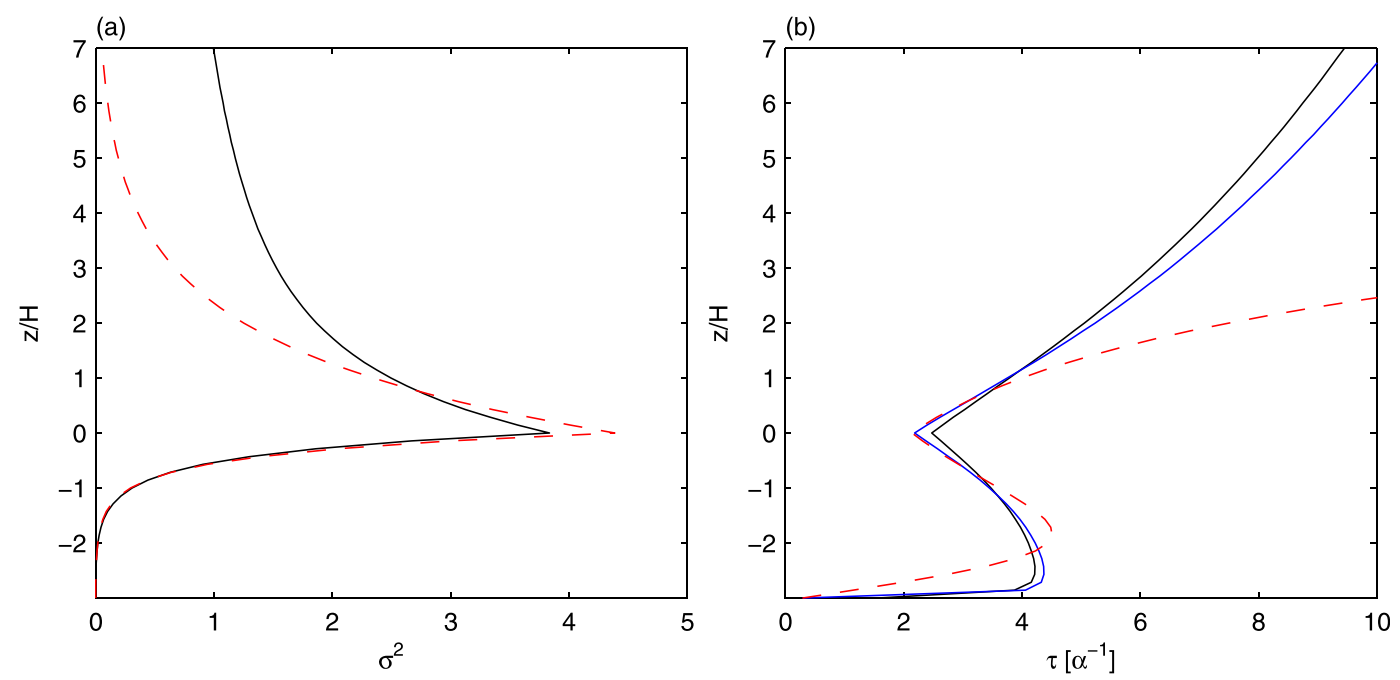

FIG. 5. As in Figs. 3c,d, but for the case with the lower boundary located at $z=-3 H$.

Ambaum 2011). Vertical variance profiles are shown in Fig. 6d.

Finally, we may complete our scenario by assuming a weakly red forcing. This can be included in our linear system by simply multiplying the spectra of the response to the white forcing by a factor $\left(\omega^{2}+\alpha_{F}^{2}\right)^{-1}$, where $\alpha_{F}^{-1}=1$ day represents a short memory of the forcing. This is plausible since even the synoptic-scale eddy forcing in the troposphere is not completely random from day to day, because the synoptic systems have their own life cycles that typically last for longer than 1 day. The results are shown in Fig. 6 by the black lines. The main effect of a weakly red forcing is the slightly slower decay of the ACF at very short lags. Additionally, the slope of the near-exponentially shaped part of the spectrum increases. This spectrum (black line in Fig. 6b) and the associated ACF (black line in Fig. 6c) are indeed close reproductions of the winter northern annular mode spectrum and ACF as shown by Osprey and Ambaum (2011); see their Figs. 2 and 3 (blue lines). This result suggests that the observed spectral and ACF structure of annular variability in the troposphere may, to some extent, be the expression of the linear effect of the frictional rigid lower boundary, together with well-separated thermal and mechanical damping rates, on the zonal-mean response to a white (or perhaps weakly red) random mechanical forcing.

\section{Discussion}

The results of the idealized tropospheric scenario presented at the end of the previous section have been shown only at $z=5 \mathrm{~km}$, which is representative of a midtropospheric level. However, the discussed characteristics of the power spectrum and, in particular, of the ACF vary with height, as was shown in Fig. 3 for the case with a single forcing located at the lower boundary and further discussed in terms of the vertical decay rates together with Fig. 4. Specifically, the relative proportion of the rapidly decaying to the slowly decaying components in the ACF decreases with height. In the idealized tropospheric scenario, an additional reason for this is that farther away from the lower boundary, the influence of the upper-level forcing dominates the solution, which is less influenced by the lower boundary, according to the exponential term in (28). By contrast, northern annular mode variability is always observed to have an equivalent barotropic structure and, thus, we shall not expect a notable change in the characteristics of its ACF with height. Consequently, the hypothesis that the observed spectral and autocorrelation structure of annular variability in the troposphere may, to some extent, be explained by the presence of a slow thermal and a fast mechanical damping time scale, needs to be complemented by, perhaps, the assumption of a possible eddy feedback. In our setup, there is no eddy feedback as the forcing is prescribed without any dependence on the state of the system, whereas eddy-zonal flow feedbacks are well known to exist in the atmosphere and in general circulation models, and different mechanisms have been proposed (e.g., Feldstein and Lee 1998; Robinson 2000; Lorenz and Hartmann 2003; Zhang et al. 2012). It is, therefore, reasonable to assume that, once the effect of the lower boundary has influenced the annular variability at midtropospheric levels as described in the previous section, eddy feedbacks may help to spread the spectral and autocorrelation characteristics over the depth of the troposphere, as tropospheric eddies generally have 

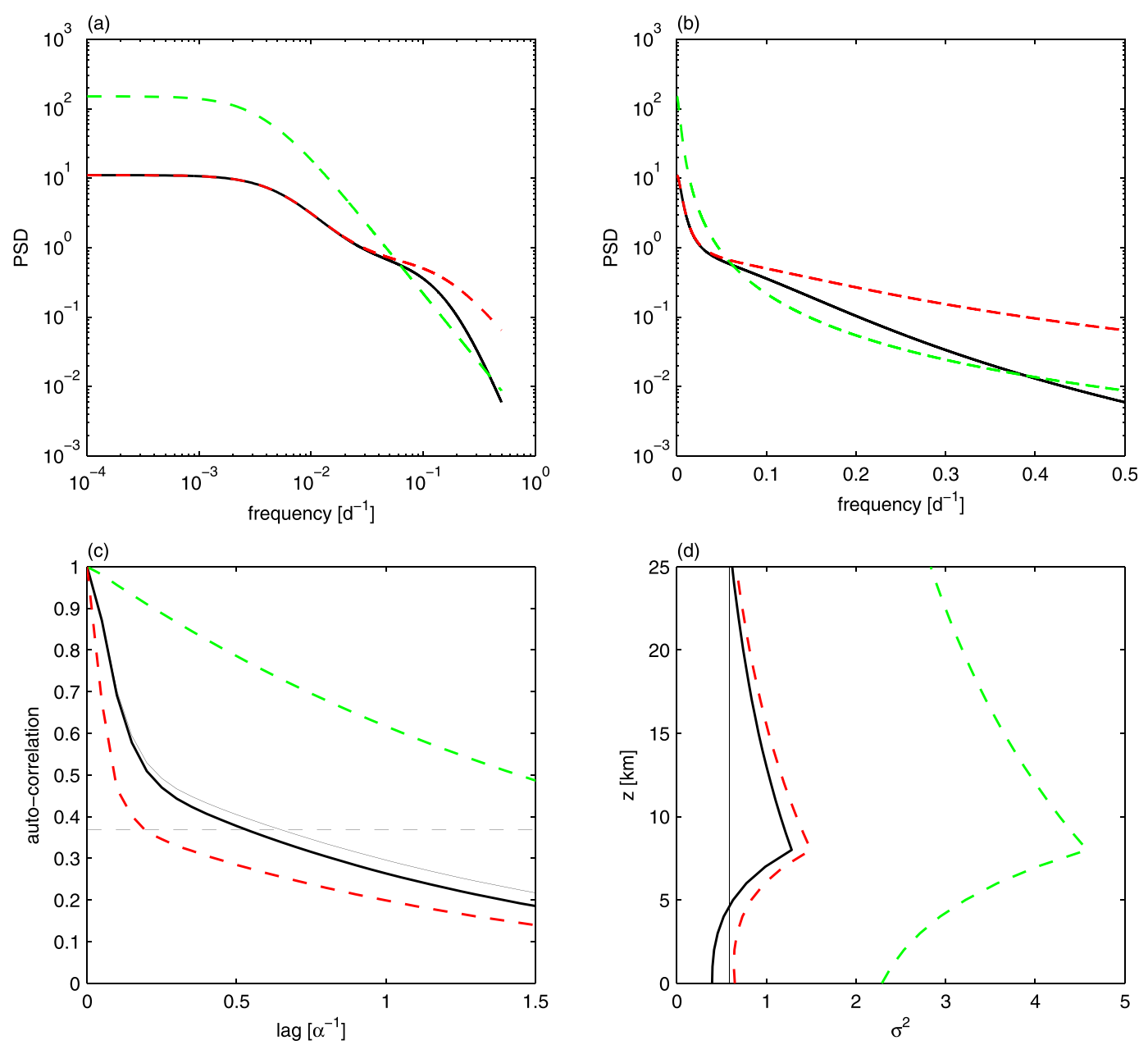

FIG. 6. For the case with one forcing located at $z=0$ and a second forcing at $z=8 \mathrm{~km}$, power spectra in (a) $\log -\log$ and (b) semilog representations, (c) ACFs, and (d) variances, for the case of an infinitely deep atmosphere (green dashed), with a frictional rigid lower boundary at $z_{b}=0$ (red dashed), and with additionally weakly red forcing (black thick). Thin black lines in (c) and (d) represent the ACF and variance, respectively, of the barotropic component. See text for details.

the effect of making the variability of the zonally averaged flow more barotropic. More specifically, anomalies in the vertical shear of the zonal flow must be expected to be quickly wiped out by baroclinic eddies, in consequence of the associated anomalous baroclinicity, whereas the barotropic component of the flow is probably less affected in such a way by eddy feedbacks.

The ACF of the barotropic component in the idealized tropospheric scenario is shown in Fig. 6c (thin black line) and is obviously very similar to the ACF of the full variability at $5 \mathrm{~km}$ (thick black line), since at this midtropospheric level the barotropic component explains almost all the variance of the flow, as seen in Fig. 6d (cf. thin and thick black lines). However, it is not clear how the additional eddy forcing that may arise from such a baroclinic feedback would, in turn, modify the autocorrelation structure of the zonal flow, including its barotropic component. Questions of this type cannot be answered by the simple approach taken in this study but need to be addressed in future research. Nevertheless, our results show that even without an eddy feedback the roots of the observed spectral and autocorrelation structure of annular variability in the troposphere may already be contained in the linear zonal-mean QG adjustment problem, when subject to a simple stochastic mechanical forcing, and in the presence of a frictional rigid lower boundary.

Regarding the horizontal structure, we may note that choosing the fourth Hough mode $(n=4)$ for our idealized tropospheric setup is sensible since this mode exhibits a pattern that is associated with exactly one zonal-mean zonal wind dipole structure per hemisphere, which can 
be interpreted as the most basic feature of the atmospheric annular modes, indicating a north-south vacillation of the midlatitude jet. Both the northern and southern annular modes in the atmosphere, however, are confined to only one hemisphere and one may thus need additional modes to obtain a representative meridional structure, and the spectra and ACFs in the previous section were shown to depend on the choice of the horizontal mode. It is, however, an intrinsic characteristic of the Hough modes that their eigenvalues and, thus, their associated length scales exhibit a paired behavior; that the values for the first two modes are relatively close to each other but rather separated from the next pair for modes 3 and 4 and another pair for modes 5 and 6 , etc.; and that the two modes that belong to the same pair can be easily combined into a pattern that is largely confined to a single hemisphere-a feature remarked on already by Dunkerton (1989); see also our Table 1. Hence, a reasonable structure that is associated with a zonal-mean zonal wind dipole in only one hemisphere can be obtained by combining the third and fourth Hough mode, and since the eigenvalue of the third mode is close to that of the fourth mode, the corresponding power spectra and ACFs for $n=3$ (not shown) are similar to the case with $n=4$ presented in the previous section.

Another aspect to be discussed concerns the spectral phase relationship between the forcing and the zonalmean response. Observed annular variability is shown to be consistent with a system of the form $d x / d t=m-x / \tau$ (Lorenz and Hartmann 2001, 2003), with the zonal index $x$, its eddy momentum forcing $m$, and a time constant $\tau$, implying a monotonically increasing phase spectrum according to an arctan function. By contrast, even the barotropic component in our idealized tropospheric scenario exhibits a different behavior, similar to the complex geopotential solution shown in Fig. $4 \mathrm{~b}$ for the case forced at a single level. Since this solution is depicted for $\hat{f}_{*}=1$, it is identical to the transfer function (at a particular level) from the forcing to the response, given by $\hat{\Phi}(\omega) / \hat{f}_{*}(\omega)$, for forcings with arbitrary amplitude and phase. From Fig. 4b, it is obvious that the phase lag of the response behind the forcing does not increase monotonically in our case but rather undergoes a slight reduction at intermediate frequencies. It is an interesting question whether this mismatch with the observed phase relationship may be reduced when an additional eddy forcing is included that may arise from a baroclinic eddy feedback as suggested above.

Finally, we note that the high-frequency part of the midtropospheric power spectrum in our idealized scenario is, certainly, not strictly exponential. For very high frequencies, it flattens out similar to a Lorentzian-shaped red noise spectrum. However, frequencies greater than $0.5 \mathrm{day}^{-1}$ do not need to be considered in a QG framework (and, thus, for annular mode variability at all) since the QG scaling arguments break down on such short time scales. And Fig. $6 \mathrm{~b}$ indicates that up to a frequency of $0.5 \mathrm{day}^{-1}$, the spectrum indeed appears nearly exponential, although it still exhibits a slight flattening beyond 0.3 day $^{-1}$; but note that a similar behavior is also found for the observed northern annular mode spectrum by close inspection of the spectrum shown by Osprey and Ambaum (2011); see their Fig. 2 (blue line) —although it is not clear whether this can be taken as a statistically significant feature.

\section{Conclusions}

To investigate the dynamical origin of the spectral and autocorrelation structure of annular variability in the troposphere, we have analyzed the characteristics of the zonal-mean geopotential variability (i.e., the quantity from which annular modes are usually derived) that arises in response to a white noise mechanical forcing, applied to a zonal-mean QG spherical atmosphere with constant static stability and uniform linear thermal damping, with the forcing reduced to a single Hough mode and concentrated at a particular level in the vertical. Analytic expressions for the power spectra of the geopotential variability have been derived, together with approximate expressions for red noise (i.e., Lorentzian shaped) spectra, which fit the true spectra in their low- and high-frequency limits. This has been done for the case of an infinitely deep atmosphere, as well as for the case that includes a frictional rigid lower boundary. The associated autocorrelation functions are obtained as the inverse Fourier transform of the corresponding power spectra (where for the true spectra the involved integral is solved numerically). Finally, the scenario has been extended to an idealized tropospheric setup that consists of two forcings, representative of a lower- and an upper-tropospheric eddy forcing. The main conclusions of our analysis are as follows:

- For the case of an infinitely deep atmosphere and for the first few Hough modes (associated with large Rossby heights), the local power spectra and autocorrelation functions, at the level of the forcing, can be well approximated by a red noise process.

- The remote power spectra and autocorrelation functions, at some distance above or below the forcing, can also be well approximated by a red noise process, provided the distance from the forcing is not larger than about one Rossby height (see Figs. 1 and 2 together with Table 1). 
- When a frictional rigid lower boundary is included, however, the approximate red noise spectra provide a generally bad fit, except at the boundary itself where a local red noise approximation, with a time scale related to the frictional time scale, fits the true spectrum well.

- Moreover, in the presence of a frictional rigid lower boundary, the power spectra and autocorrelation functions exhibit distinct behavior at low and high frequencies. Specifically, the high-frequency part of the power spectrum changes into a near-exponential behavior as opposed to a near-red noise behavior without the lower boundary. The associated autocorrelation functions show a sharp transition from a rapid decay at short lags to a slow decay at long lags (see Figs. 6b and $6 \mathrm{c}$ for the case of the idealized tropospheric setup).

- These fast and slow components seen in the power spectra and autocorrelation functions can be identified as (i) the thermally undamped component that arises from the interaction between the mechanical forcing and the frictional boundary and (ii) the interaction between the thermal damping, which acts on the produced anomalies, and the frictional boundary.

- Finally, the sharpness of the transition between the fast and the slow components increases the more the thermal and frictional damping time scales are separated from each other (not shown).

Since the spectral and autocorrelation structure of observed annular variability exhibits the same distinct time-scale behavior (Lorenz and Hartmann 2001, 2003; Osprey and Ambaum 2011), the above results suggest that these characteristics may, to some extent, be intrinsic to the linear zonal-mean response problem. The vertical structure of the variability as well as the phase relationship between the forcing and the response, however, do not fully agree with observations. It is likely that (presumably baroclinic) eddy feedbacks could, at least partly, resolve these discrepancies, although such questions lie beyond the scope of this study and should be the subject of future research. At this point, it is noteworthy that a complementary study by Zurita-Gotor et al. (2014), investigating annular variability in a $\beta$-plane two-layer model, indicates that a similar distinct time-scale behavior may, in their simple model, also be obtained from eddy-zonal flow feedbacks alone, without any contributions from the dynamics of the zonal-mean circulation. Additionally, although our results imply a contribution to the distinct time-scale behavior from linear dynamics (but note that eddy feedbacks may introduce some nonlinearity), this does not rule out the possibility of contributions from nonlinear low-order dynamical chaos, as suggested by the hypothesis of Osprey and Ambaum
(2011). Observational evidence for nonlinear aspects of the driving of teleconnections in general is also put forward in Athanasiadis and Ambaum (2009), although the relevance of their results for the variability of the zonal-mean flow is not entirely obvious, since they are only concerned with two-dimensional horizontal teleconnection patterns. Finally, whereas such internal dynamics are probably most relevant to the variability on intraseasonal time scales, impacts of slow external forcings (e.g., Keeley et al. 2009) must be expected to play an important role in driving decadal- and longer-time-scale fluctuations of annular variability, and these may indeed also influence the shape of the autocorrelation function, even at lags of just a few weeks, as the autocorrelation at a given lag depends on variability at all frequencies.

Acknowledgments. TK and RJG are grateful to support from GEOMAR during the time this work was carried out. The authors also thank three anonymous reviewers for their helpful comments.

\section{REFERENCES}

Andrews, D. G., J. R. Holton, and C. B. Leovy, 1987: Middle Atmosphere Dynamics. Academic Press, 489 pp.

Athanasiadis, P. J., and M. H. P. Ambaum, 2009: Linear contributions of different time scales to teleconnectivity. J. Climate, 22, 3720-3728, doi:10.1175/2009JCLI2707.1.

Baldwin, M. P., and T. J. Dunkerton, 2001: Stratospheric harbingers of anomalous weather regimes. Science, 294, 581-584, doi:10.1126/science.1063315.

Brayshaw, D. J., B. Hoskins, and M. Blackburn, 2009: The basic ingredients of the North Atlantic storm track. Part I: Landsea contrast and orography. J. Atmos. Sci., 66, 2539-2558, doi:10.1175/2009JAS3078.1.

Dunkerton, T. J., 1989: Body force circulations in a compressible atmosphere: Key concepts. Pure Appl. Geophys., 130, 243 262, doi:10.1007/BF00874458.

Feldstein, S. B., 2000: The timescale, power spectra, and climate noise properties of teleconnection patterns. J. Climate, 13, 4430-4440, doi:10.1175/1520-0442(2000)013<4430:TTPSAC >2.0.CO;2.

— , and S. Lee, 1998: Is the atmospheric zonal index driven by an eddy feedback? J. Atmos. Sci., 55, 3077-3086, doi:10.1175/ 1520-0469(1998)055<3077:ITAZID > 2.0.CO;2.

Garcia, R. R., 1987: On the mean meridional circulation of the middle atmosphere. J. Atmos. Sci., 44, 3599-3609, doi:10.1175/ 1520-0469(1987)044<3599:OTMMCO >2.0.CO;2.

Haynes, P. H., and T. G. Shepherd, 1989: The importance of surface pressure changes in the response of the atmosphere to zonally-symmetric thermal and mechanical forcing. Quart. J. Roy. Meteor. Soc., 115, 1181-1208, doi:10.1002/ qj.49711549002.

_ C. J. Marks, M. E. McIntyre, T. G. Shepherd, and K. P. Shine, 1991: On the "downward control" of extratropical diabatic circulations by eddy-induced mean zonal forces. J. Atmos. Sci., 48, 651-678, doi:10.1175/1520-0469(1991)048<0651: OTCOED $>2.0 . \mathrm{CO} ; 2$.

Held, I. M., and T. Schneider, 1999: The surface branch of the zonally averaged mass transport circulation in the troposphere. J. Atmos. 
Sci., 56, 1688-1697, doi:10.1175/1520-0469(1999)056<1688: TSBOTZ $>2.0 . \mathrm{CO} ; 2$.

Hoerling, M. P., J. W. Hurrell, and T. Xu, 2001: Tropical origins for recent North Atlantic climate change. Science, 292, 90-92, doi:10.1126/science.1058582.

Hurrell, J. W., 1995: Decadal trends in the North Atlantic Oscillation: Regional temperatures and precipitation. Science, 269, 676-679, doi:10.1126/science.269.5224.676.

Keeley, S. P. E., R. T. Sutton, and L. C. Shaffrey, 2009: Does the North Atlantic Oscillation show unusual persistence on intraseasonal timescales? Geophys. Res. Lett., 36, 367, doi:10.1029/ 2009 GL040367.

Longuet-Higgins, M. S., 1968: The eigenfunctions of Laplace's tidal equations over a sphere. Philos. Trans. Roy. Soc. London, A262, 511-607, doi:10.1098/rsta.1968.0003.

Lorenz, D. J., and D. L. Hartmann, 2001: Eddy-zonal flow feedback in the Southern Hemisphere. J. Atmos. Sci., 58, 3312-3327, doi:10.1175/1520-0469(2001)058<3312:EZFFIT >2.0.CO;2.

$\longrightarrow$, and —, 2003: Eddy-zonal flow feedback in the Northern Hemisphere winter. J. Climate, 16, 1212-1227, doi:10.1175/ 1520-0442(2003)16<1212:EFFITN>2.0.CO;2.

Osprey, S. M., and M. H. P. Ambaum, 2011: Evidence for the chaotic origin of Northern Annular Mode variability. Geophys. Res. Lett., 38, L15702, doi:10.1029/2011GL048181.
Plumb, R. A., 1982: Zonally symmetric Hough modes and meridional circulations in the middle atmosphere. J. Atmos. Sci., 39, 983-991.

Robinson, W. A., 2000: A baroclinic mechanism for the eddy feedback on the zonal index. J. Atmos. Sci., 57, 415-422, doi:10.1175/1520-0469(2000)057<0415:ABMFTE > 2.0.CO;2.

Stephenson, D. B., V. Pavan, and R. Bojariu, 2000: Is the North Atlantic Oscillation a random walk? Int. J. Climatol., 20, 1-18, doi:10.1002/(SICI)1097-0088(200001)20:1<1:: AID-JOC456>3.0.CO;2-P.

Thompson, D. W. J., and J. M. Wallace, 1998: The Arctic Oscillation signature in the wintertime geopotential height and temperature fields. Geophys. Res. Lett., 25, 1297-1300, doi:10.1029/98GL00950.

Wallace, J. M., 2000: North Atlantic Oscillation/annular mode: Two paradigms-One phenomenon. Quart. J. Roy. Meteor. Soc., 126, 791-805, doi:10.1256/smsqj.56401.

Zhang, Y., X.-Q. Yang, and Y. Nie, 2012: Annular mode-like variation in a multilayer quasigeostrophic model. J. Atmos. Sci., 69, 2940-2958, doi:10.1175/JAS-D-11-0214.1.

Zurita-Gotor, P., J. Blanco-Fuentes, and E. Gerber, 2014: The impact of baroclinic eddy feedback on the persistence of jet variability in the two-layer model. J. Atmos. Sci., 71, 410-429, doi:10.1175/JAS-D-13-0102.1. 